\title{
Collision tumor: pigmented Bowen's disease and seborrheic keratosis*
}

\author{
Karen de Almeida Pinto Fernandes ${ }^{1}$, Diana Carolina Salamanca Martinez ${ }^{1}$, Aretha Brito Nobre ${ }^{2}$, \\ Gabriella Campos-do-Carmo ${ }^{1}$
}

DOI: http:/ / dx.doi.org/10.1590/abd1806-4841.20187117

\begin{abstract}
Bowen's disease is a type of squamous cell carcinoma in situ of the skin. The pigmented form is rare and represents less than $2 \%$ of cases. We report a case of a 74-year-old black man with a blackened and asymptomatic leg injury for about 6 months. Clinically, and under dermatoscopy, the injury was suggestive of melanoma or melanoacanthoma, and a definitive diagnosis was made by histopathological examination.
\end{abstract}

Keywords: Bowen's disease; Melanoma; Keratosis, seborrheic

\section{INTRODUCTION}

Pigmented Bowen's disease is a rare squamous cell carcinoma in situ, most prevalent in patients that are male and older than 70 years of age. ${ }^{1}$ It was first described in 1912 by J. T. Bowen and presents the potential to develop into an invasive tumor. ${ }^{2,3}$ Clinically, it manifests as a well-defined, asymptomatic and slow-growing erythematous plaque with a scaly or crusted surface. ${ }^{2,3}$ The main risk factors are sun exposure, arsenic intoxication, immunosuppression, human papillomavirus (HPV) infection, trauma and ionizing radiation. ${ }^{1-4}$ The pigmented variant is rare; according to Ragi et al., in a 1988 review of 420 cases, only seven (1.7\%) were pigmented Bowen's disease. ${ }^{1,3,4}$ This form is most prevalent in black men around 70 years of age and in photoprotected areas, which differs from the non-pigmented form. ${ }^{5}$ The main differential diagnoses of the pigmented form include seborrheic keratosis, melanoma, pigmented actinic keratosis, solar lentigo, pigmented basal cell carcinoma, melanocytic nevus, and blue nevus. ${ }^{1,3,5-7}$ Furthermore, some cases occur in association with seborrheic keratosis. ${ }^{4}$ The histopathological exam of pigmented Bowen's disease shows epidermal dysplasia, characterized by irregular keratinocyte maturation, abnormal keratinization and disorganized architecture. Hyperkeratosis, parakeratosis and acanthosis may be present. Nuclear atypia and mitosis are frequent. The dermis does not present invasion and often contains a dense lymphocytic or histiocytic infiltrate. Moreover, in this pigmented form, we find an abundance of melanocytes in the epidermis. ${ }^{1,3,5,7,8}$ The choice of treatment depends on factors such as localization, size and number of lesions as well as the patient's comorbidities. Several option have been described, such as surgical excision, topical 5-fluorouracil, topical imiquimod, cryotherapy, curettage and electrocoagulation, radiotherapy, laser and photodynamic therapy., ${ }^{2,3}$

\footnotetext{
Received 14 March 2017.

Accepted 28 August 2017.

* Work conducted at the Instituto Nacional do Câncer, Rio de Janeiro (RJ), Brazil. Financial support: None. Conflict of interest: None.

Oncological Dermatology Service, Instituto Nacional do Câncer, Rio de Janeiro (RJ), Brazil. Division of Pathology, Instituto Nacional do Câncer, Rio de Janeiro (RJ), Brazil.
}

\section{MAILING AdDRESS:}

Karen de Almeida Pinto Fernandes

E-mail: karenfernandes22gf@hotmail.com

(C)2018 by Anais Brasileiros de Dermatologia 


\section{CASE REPORT}

A 74-year-old, black, male patient, native of Rio de Janeiro, sought dermatological treatment due to the emergence of black pigmentation, over the previous six months, on the edge of a lesion that had existed on his right leg for more than 20 years. An examination revealed a brownish plaque on the lateral face of the right leg, suggestive of seborrheic keratosis, and a blackened, papular lesion with irregular borders, approximately $5 \mathrm{~mm}$ in size, at the edge of the plaque, suggestive of melanoma or melanoacanthoma, which would constitute a collision tumor (Figure 1). The patient denied any associated symptoms. His prior pathological history included a prostate adenocarcinoma since 2012, and he denied a family history of cutaneous neoplasia. Dermatoscopy of the plaque showed a cerebriform pattern in the upper region of the lesion, central crust, and a fingerprint pattern on the border, findings compatible with seborrheic keratosis (Figure 2). The dermatoscopy of the papular lesion revealed a blackened blotch, pigment network in the lower portion, rosette, pseudopod and bluish-gray veil-criteria suspicious for melanoma (Figure 3). The histopathology confirmed hyperkeratosis with papillomatosis, acanthosis, and hyperpigmentation of basal keratinocytes, constituting seborrheic keratosis, in association with intraepithelial neoplasia of pigmented and depolarized keratinocytes, constituting pigmented epidermoid carcinoma in situ. Perivascular mononuclear inflammatory infiltrate was also observed in the upper dermis (Figure 4). A higher magnification revealed mitotic figures in the upper third of the epidermis, as well as dilated blood vessels in the dermis (Figure 5).

\section{DISCUSSION}

In a series of 52 cases of pigmented Bowen's disease, some specific dermatoscopic patterns were found, such as grayish-brown spots in linear arrangement and glomerular vessels. Like other pig-

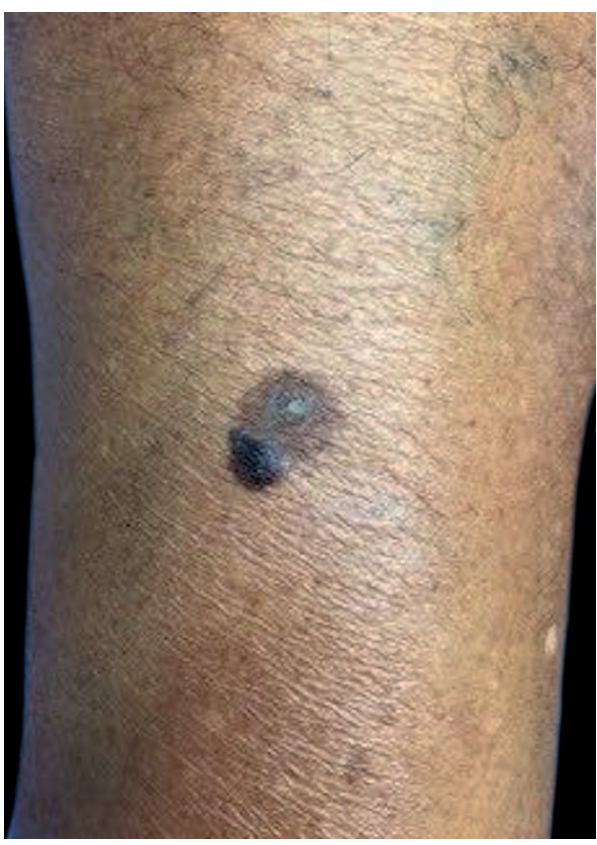

Figure 1:

A brownish plaque on the lateral face of the right leg, suggestive of seborrheic keratosis, and a blackened, papular lesion with irregular borders at the edge of the plaque, suggestive of melanoacanthoma or melanoma mented lesions, pigmented Bowen's disease may have various clinical and dermoscopic presentations. Widely observed patterns in this study were areas without structures $(n=25)$ and spots associated with areas without structures $(n=18)$. Furthermore, according to the histopathology, $17.3 \%$ of cases $(n=9)$ were associated with seborrheic keratosis. ${ }^{4}$ In our case, the blackened blotch and the area of bluish-gray veil made the lesion highly suspicious for melanoma associated or colliding with preexisting seborrheic keratosis. We also proposed the hypothesis that the blackened part of the lesion was a melanoacanthoma, which can also mimic melanoma. ${ }^{9}$ The term 'melanoacanthoma' denotes a rare and intensely pigmented variant of seborrheic keratosis. The term was adopted by Mishima and Pinkus in 1960 to refer to a benign, pigmented lesion secondary to the proliferation of melanocytes and keratinocytes in the

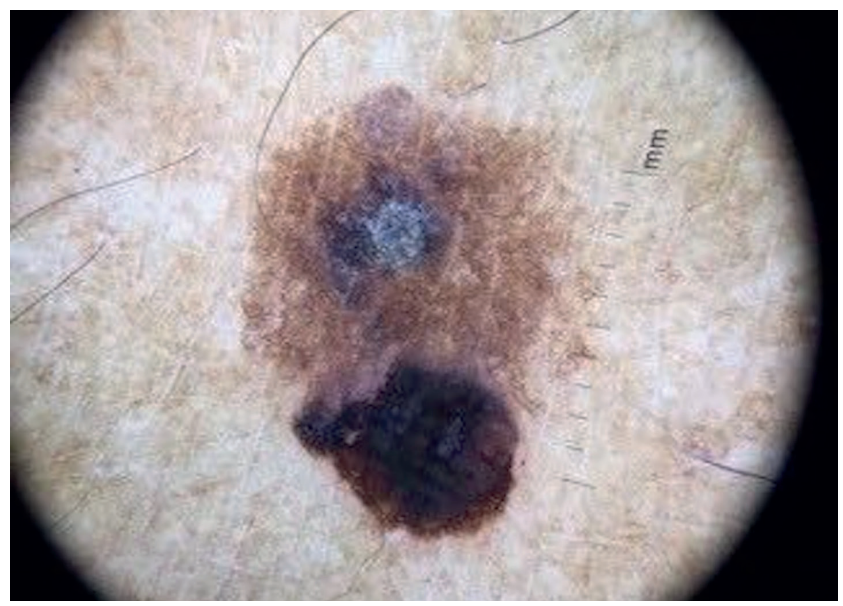

FiguRE 2: Dermatoscopy of the plaque lesion: cerebriform pattern in the upper region of the lesion, central crust, and fingerprint pattern on the border-findings compatible with seborrheic keratosis

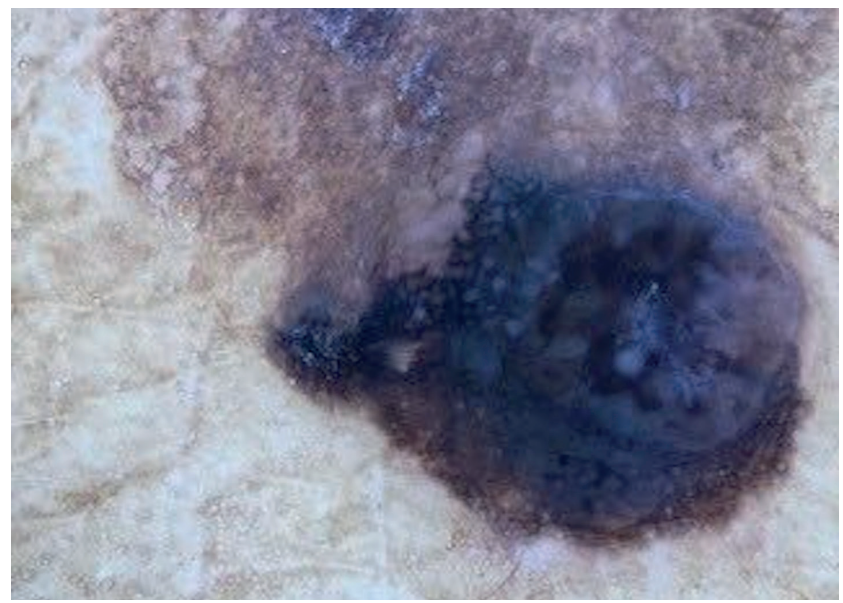

FIgURE 3: Dermatoscopy of the papular lesion at higher magnification, showing a melanoma-like lesion with blackened blotch, pigment network in the lower region, rosette, pseudopod and bluish-gray veil 


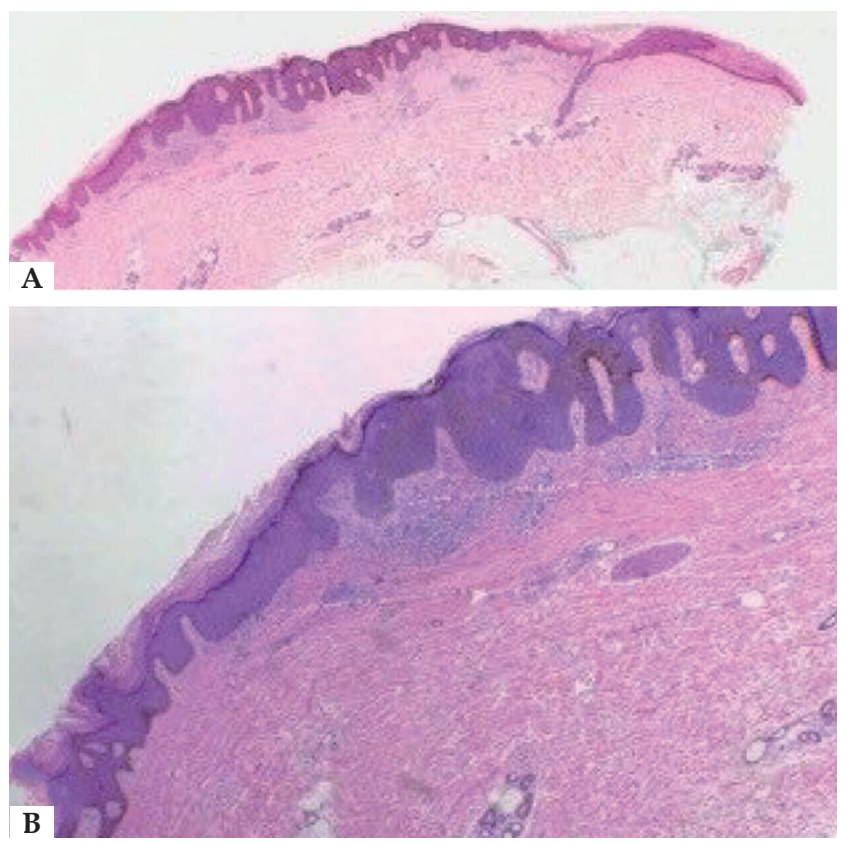

Figure 4: A - On the left, area of hyperkeratosis with papillomatosis, acanthosis and hyperpigmentation of basal keratinocytes, constituting seborrheic keratosis; on the right, intraepithelial neoplasia of pigmented and depolarized keratinocytes, constituting pigmented epidermoid carcinoma in situ (Hematoxylin \& eosin, x2); B - mononuclear perivascular inflammatory infiltrate in the upper dermis (Hematoxylin \& eosin, x10)

epidermal layers. Melanoacanthoma manifests as a slow-growing, usually solitary lesion located on the head, neck or trunk of older people, melanoma being its main differential diagnosis. ${ }^{9}$ Collision tumors are characterized by the coexistence of two neoplasms at the

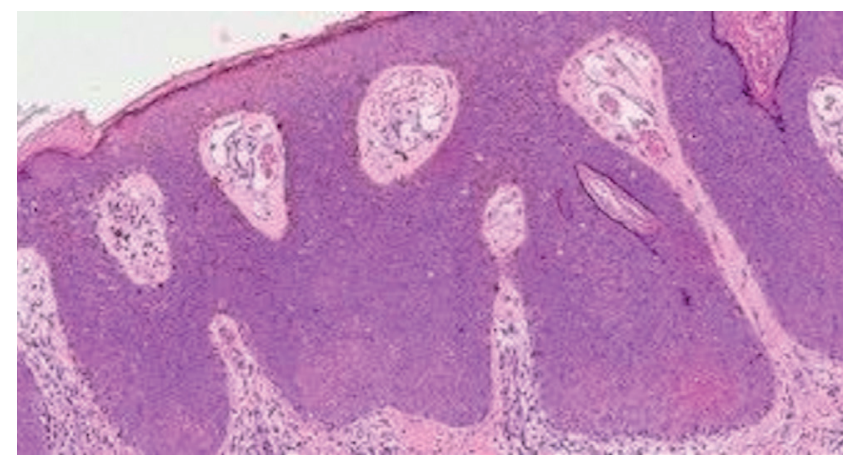

FIGURE 5: Hyperkeratosis, foci of parakeratosis, acanthosis, and atypical keratinocytes, sometimes hyperpigmented, occupy the entire thickness of the epidermis; mitotic figures are noted in the upper third of the epidermis; the dermis contains dilated vessels and mononuclear perivascular inflammatory infiltrate with melanophages (Hematoxylin \& eosin, x20)

same anatomical site, and their pathogenesis remains contentious. In these cases, prognosis and treatment are dictated by the tumor of greater severity. ${ }^{10}$ Dermatoscopy is an auxiliary tool in the diagnosis of pigmented lesions. In the case presented here, the blackened portion of the lesion showed criteria suspicious for melanoma. It should be noted that we found no reports in the literature of this atypical dermatoscopic presentation of pigmented Bowen's disease, which makes this case even rarer. The histopathology, reference standard in cutaneous diagnoses, revealed the definitive diagnosis in this unusual case of pigmented Bowen's disease associated with seborrheic keratosis.

\section{REFERENCES}

1. Mota AN, Piñeiro-Maceira J, Alves Mde F, Tarazona MJ. Pigmented Bowen's disease. An Bras Dermatol. 2014;89:825-7.

2. Neubert T, Lehmann P. Bowen's disease-a review of newer treatment options. Ther Clin Risk Manag. 2008:4:1085-95.

3. Lee JW, Hur J, Yeo KY, Yu HJ, Kim JS. A case of pigmented Bowen's disease. Ann Dermatol. 2009;21:249-51.

4. Cameron A, Rosendahl C, Tschandl P, Riedl E, Kittler H. Dermatoscopy of pigmented Bowen's disease. J Am Acad Dermatol. 2010;62:597-604.

5. Krishnan R, Lewis A, Orengo IF, Rosen T. Pigmented Bowen's disease (squamous cell carcinoma in situ): a mimic of malignant melanoma. Dermatol Surg. 2001;27:673-4.
6. Hernández-Gil J, Fernández-Pugnaire MA, Serrano-Falcón C, Serrano-Ortega S. Clinical and dermoscopic features of pigmented Bowen disease. Actas Dermosifiliogr. 2008;99:419-20.

7. Firooz A, Farsi N, Rashighi-Firoozabadi M, Gorouhi F. Pigmented Bowen's disease of the finger mimicking malignant melanoma. Arch Iran Med. 2007;10:255-7.

8. Namiki T, IchiyamaS, Funasaka Y, Ito M, Kanzaki A, Miura K, Nojima K, Saeki H, Yokozeki H, Tanaka M. Dermoscopy of pigmented papillated Bowen disease: A report of two cases. J Dermatol. 2017;44:e23-e24.

9. Shankar V, Nandi J, Ghosh K, Ghosh S. Giant melanoacanthoma mimicking malignant melanoma. Indian J Dermatol. 2011;56:79-81.

10. Medeiros PM, Alves NR, Silva CC, Faria PC, Barcaui CB, Piñeiro-Maceira J. Collision of malignant neoplasms of the skin: basosquamous cell carcinoma associated with melanoma. An Bras Dermatol. 2015;90:39-42.

\footnotetext{
Aretha Brito Nobre

ORCID 0000-0002-7177-9838

Collecting, analysis and interpretation of data

Gabriella Campos-do-Carmo

ORCID 0000-0003-2258-5823

Intellectual participation in propaedeutic and/or therapeutic conduct of studied cases
}

How to cite this article: Fernandes KAP, Martinez DCS, Nobre AB, Campos-do-Carmo G. Collision tumor: pigmented Bowen's disease and seborrheic keratosis. An Bras Dermatol. 2018;93(5):737-9. 